\title{
Accretion of Neutral Hydrogen and Helium onto a Neutron Star. The X-ray and Gamma-Ray Radiation of Cool Neutron Stars
}

\author{
E. M. Kantor and A. I. Tsygan \\ Ioffe Physicotechnical Institute, Russian Academy of Sciences, \\ Politekhnicheskaya ul.26, St. Petersburg, 194021 Russia \\ Received October 10, 2004; in final form, September 7, 2005
}

\begin{abstract}
The accretion of neutral gas (hydrogen and helium) onto a neutron star is studied. The gas is gravitationally captured into the magnetosphere of the star, where it is ionized by thermal radiation from the stellar surface and accelerated by the electric field at the light cylinder and in a tube of open magnetic lines. The particle acceleration at the light cylinder generates gamma-rays, some of which move towards the star and heat its polar regions, resulting in the emission of X-rays. Our calculations of the model parameters of the X-ray and gamma-ray radiation indicate that the radiation intensities should be sufficient to be observed.
\end{abstract}

PACS numbers : $97.60 . \mathrm{J}$

DOI: $10.1134 / \mathrm{S} 1063772906020089$

\section{INTRODUCTION}

The capture of charged particles of the interstellar medium by the gravitational fields of neutron stars is hindered by the fact that these particles are repelled by the magnetic-dipole radiation of the neutron star and the flow of charged relativistic particles with its frozen-in magnetic field. It is easy to show (see, for example, [1]) that the accretion of interstellar plasma is impossible if the rate of rotational-energy loss of the neutron star exceeds $6 \times 10^{25} \mathrm{erg} / \mathrm{s}$ (for a number density of the interstellar plasma $n=1 \mathrm{~cm}^{-3}$ and the velocity of the star $V=2 \times 10^{7} \mathrm{~cm} / \mathrm{s}$ ). For typical rotational-energy loss rates $\left(10^{31} \mathrm{erg} / \mathrm{s}\right)$, only neutral particles can be captured, since they do not interact with electromagnetic radiation or the relativistic flow. The surface radiation of hot, young neutron stars efficiently ionizes the surrounding neutral atoms. However, cool neutron stars may be surrounded by neutral interstellar gas (which consists of $90 \%$ hydrogen and $10 \%$ helium atoms), which can be captured by their gravitational fields and ionized in their magnetospheres by thermal radiation from their surfaces (in the vicinity of the light-cylinder radius $R_{\mathrm{LC}}=c / \Omega$, where $\Omega$ is the angular velocity of the stellar rotation).

The possibility of accreting neutral hydrogen from the interstellar medium onto a cool (surface temperature $T_{\mathrm{S}} \approx 3 \times 10^{4} \mathrm{~K}$ ) neutron star was considered in 1977 by Tsygan [2], who concluded that the accretion of neutral hydrogen is required in order for the radio pulsar mechanism to operate. The accretion of neutral hydrogen onto a radio pulsar was again considered two years later (in 1979) by Wright [3]. Of course, we now understand that the accretion of neutral hydrogen is not required for the operation of most radio pulsars. It was shown in $[2,4]$ that the accretion of interstellar neutral hydrogen onto cool, switched-off radio pulsars results in two effects: (1) X-ray emission due to heating of the polar regions of the stars and (2) the generation of gammaray radiation in the region of the light cylinder. With $T_{\mathrm{S}} \approx 3 \times 10^{4} \mathrm{~K}$ (for a rotational period $P=1 \mathrm{~s}$ ), neutral hydrogen in this region is ionized by the thermal radiation of the neutron star. Note that the polar regions are heated by electrons and protons accelerated by the electric field in the vicinity of open magnetic lines. This mechanism is much more efficient than heating by the gravitational energy of protons.

We now wish to turn our attention to the accretion of neutral helium. Although it comprises only $10 \%$ of the total number of atoms, its ionization energy $\left(I_{0}=24.6 \mathrm{eV}\right)$ is a factor of 1.8 higher than that of hydrogen $\left(I_{0}=13.6 \mathrm{eV}\right)$, and we can therefore consider neutron stars with surface temperatures that are higher by approximately this same factor $\left(T_{\mathrm{S}} \approx 5 \times 10^{4} \mathrm{~K}\right)$. Calculations of the cooling of neutron stars indicate (see, for example, [5]) that their surface temperatures can decrease to $5 \times 10^{4} \mathrm{~K}$ over a time of $10^{6}-10^{7} \mathrm{yr}[5]$ if no efficient heating mechanisms operate (internal friction [6], etc.). 


\section{THE IONIZATION RATE \\ IN THE MAGNETOSPHERE}

When the surface temperature of a star is lower than the ionization energy, ionization is brought about by photons in the Wien range of the spectrum with energies exceeding the ionization energy. The number density of these photons is

$$
n_{\mathrm{ph}}=\left(\frac{a}{r}\right)^{2} \frac{k^{3} T_{\mathrm{S}}^{3}}{4 \pi^{2} c^{3} \hbar^{3}}\left(\frac{I_{0}}{k T_{\mathrm{S}}}\right) \exp \left(-\frac{I_{0}}{k T_{\mathrm{S}}}\right),
$$

where $I_{0}$ is the ionization energy of a given atom $\left(I_{0}=13.6 \mathrm{eV}\right.$ for hydrogen and $I_{0}=24.6 \mathrm{eV}$ for he- lium), and $T_{\mathrm{S}}$ and $a$ the surface temperature and radius of the star.

The cross sections for ionization by photons with energies only slightly exceeding the ionization thresholds are $\sigma_{\mathrm{ph}}=6 \times 10^{-18} \mathrm{~cm}^{2}$ and $\sigma_{\mathrm{ph}}=7 \times 10^{-18} \mathrm{~cm}^{2}$ for hydrogen and helium, respectively.

We constructed a numerical model for the accretion of neutral hydrogen and helium onto a neutron star. We assumed that the particles move along Keplerian trajectories without collisions. In the rest frame of the star, the trajectory of a particle is hyperbolic [7]:

$$
r=\frac{\rho^{2} V_{\infty}^{2} / G M}{1+\cos \left[\varphi-\arccos \left(-\frac{1}{\sqrt{1+\rho^{2} V_{\infty}^{4} / G^{2} M^{2}}}\right)\right] \sqrt{1+\frac{\rho^{2} V_{\infty}^{4}}{G^{2} M^{2}}}}
$$

Here, $r$ is the distance from the star to the particle, $\varphi$ the angle between the initial (when the particle is at infinity) and the current direction toward the particle, $\rho$ the impact parameter, $V_{\infty}$ the velocity of the neutral particle at infinity (equal to the velocity of the star relative to the interstellar gas), and $M$ the mass of the star.

The condition that the flow be continuous yields a number density for the neutral gas at the point with coordinates $(r, \varphi)$

$$
n(r, \varphi)=n_{\infty} \frac{\rho V_{\infty} \sqrt{\frac{1}{r^{2}}\left(\frac{\partial r}{\partial \varphi}\right)_{\rho}^{2}+1}}{r \sin \varphi V(r)\left(\frac{\partial r}{\partial \rho}\right)_{\varphi}} e^{-\lambda}
$$

Here, $n_{\infty}$ is the number density of the neutral at infinity, $V(r)$ the velocity of the neutral at a given point, $e^{-\lambda}$ a factor taking into account the "eating away" of the neutral atoms by the stellar radiation. $\lambda$ is the number of mean-free paths (relative to ionization), and is specified by the following integral along the trajectory:

$$
\begin{gathered}
\lambda=n_{\mathrm{PhSurf}} c \\
\times 4 \pi a^{2} \sigma_{\mathrm{ph}} \int_{0}^{\varphi} \frac{1}{4 \pi r^{2}} \frac{\sqrt{\left(\partial r / \partial \varphi^{\prime}\right)_{\rho}^{2}+r^{2}} d \varphi^{\prime}}{\sqrt{V_{\infty}^{2}+2 G M / r}},
\end{gathered}
$$

where $n_{\mathrm{PhSurf}}$ is the photon number density near the stellar surface.
The ionization rate $\left(1 / \mathrm{cm}^{3} \mathrm{~s}\right)$ is specified by the relation

$$
d N_{\mathrm{ion}} / d t=n_{\mathrm{ph}} c \sigma_{\mathrm{ph}} n(r, \varphi) .
$$

Recombination can be neglected, since the number density of ionized particles is low. We will not consider the ripping off of the second electron from the helium ion, since, when the first electron is ripped off, the resulting ion rapidly reaches high gammafactors in the electric field of the star, causing the cross section for its interaction with thermal photons to decrease sharply. We obtained the following results using this model. Figures 1-6 present curves corresponding to equal ionization rates and equal fractions of surviving neutrals for helium and hydrogen (numerical values are given for a number density of interstellar hydrogen of $1 \mathrm{~cm}^{-3}$ ).

\section{THE GENERATION \\ OF X-RAY AND GAMMA-RAY RADIATION}

Particles ionized in the neutron-star magnetosphere in the region of open magnetic lines will be accelerated along these lines by the electric field. The electrostatic potential away from the surface of the star is specified by the Arons-Scharlemann term [8]:

$$
\begin{aligned}
& \Phi\left(\eta_{\text {ion }}, \xi\right) \\
& =\frac{3}{8} B a\left(\frac{\Omega a}{c}\right)^{5 / 2} \sqrt{\eta_{\text {ion }}} \xi\left(1-\xi^{2}\right) \sin \chi \cos \phi \text {. }
\end{aligned}
$$




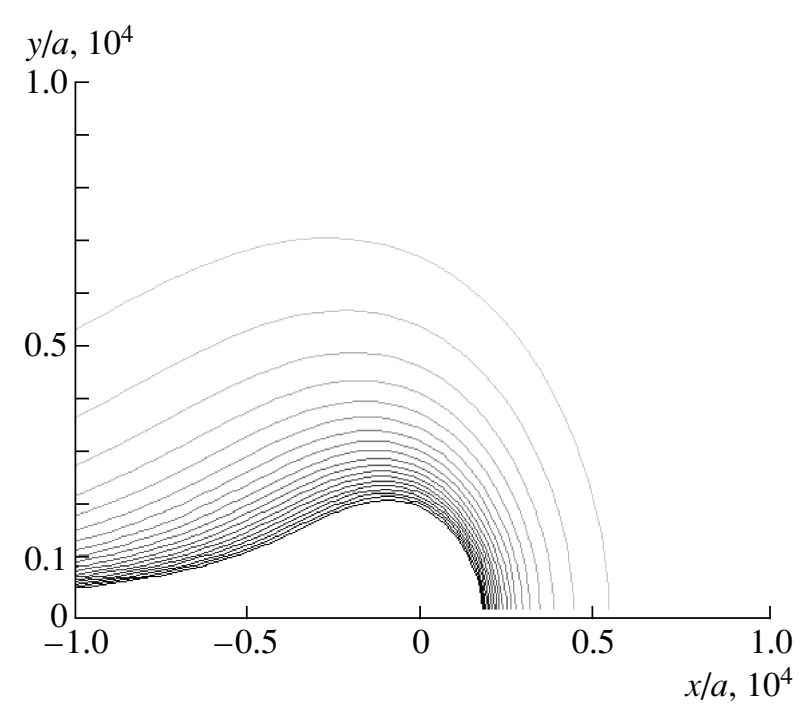

Fig. 1. Contours of equal hydrogen ionization rate. Spatial coordinates normalized to the radius of the neutron star, $10^{6} \mathrm{~cm}$, are plotted along the coordinate axes. The neutron star is located at $(0,0)$ and is moving to the right with the velocity $2 \times 10^{7} \mathrm{~cm} / \mathrm{s}$. The surface temperature of the star is $0.3 \times 10^{5} \mathrm{~K}$. The ionization rate varies from $0.012 \mathrm{~cm}^{-3} \mathrm{~s}^{-1}$ for the lightest contour to $0.345 \mathrm{~cm}^{-3} \mathrm{~s}^{-1}$ for the darkest.

This potential can be expressed in terms of the magnetic field at the light cylinder $B_{\mathrm{LC}}$ :

$$
\begin{gathered}
\Phi\left(r_{\text {ion }}, \xi\right) \\
=\frac{3}{8} B_{\mathrm{LC}}\left(\frac{c}{\Omega}\right)^{1 / 2} \sqrt{r_{\text {ion }}} \xi\left(1-\xi^{2}\right) \sin \chi \cos \phi .
\end{gathered}
$$

Particles with charge of one sign will be accelerated towards the star and heat a spot in the polar region, while particles of the other sign will be accelerated from the star. Ions will be accelerated towards the star in the part of the tube where $\cos \phi>0$, while electrons will be accelerated toward the region where $\cos \phi<0$. Figure 7 presents the results of our model calculations for the power that goes into heating the polar spots for various surface temperatures. Numerical values are given for a magnetic field near the stellar surface $10^{12} \mathrm{G}$ and a rotational period of $1 \mathrm{~s}$.

If we assume that all the energy of the particles entering the polar regions goes into the blackbody radiation of the spot, then, with $T_{\mathrm{S}}=0.3 \times 10^{5} \mathrm{~K}$, the temperature of the spot will be roughly $T=1.5 \times 10^{6} \mathrm{~K}$.

We will show that polar spots with such temperatures cannot contribute substantially to ionization at the light cylinder. For a spot temperature much higher than the ionization energy, only photons with energies of the order of the ionization energy contribute substantially to the ionization. In this region, the number

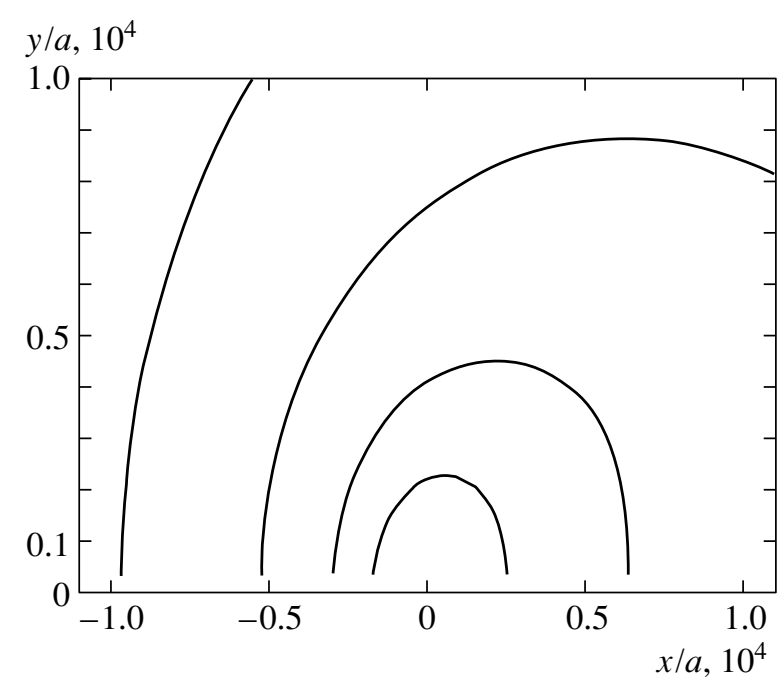

Fig. 2. Contours of equal fractions of surviving hydrogen neutrals. Spatial coordinates normalized to the radius of the neutron star, $10^{6} \mathrm{~cm}$, are plotted along the coordinate axes. The neutron star is located at $(0,0)$ and is moving to the right with the velocity $2 \times 10^{7} \mathrm{~cm} / \mathrm{s}$. The surface temperature of the star is $0.3 \times 10^{5} \mathrm{~K}$. The fraction of surviving neutrals varies from 0.3 to 0.6 .

of photons increases as $\bar{\omega}$, while the ionization cross section decreases as $\bar{\omega}^{-3.5}$. Therefore, the number of such photons radiated by the spots per unit time can be estimated as the number of photons radiated by the spots in the energy interval from $I_{0}$ to $2 I_{0}\left(I_{0}\right.$ is the ionization energy for hydrogen):

$$
\begin{aligned}
N & \approx \frac{45}{\pi^{4}} \frac{\sigma T^{4}}{k T}\left(\frac{I_{0}}{k T}\right)^{2} \pi \Theta_{0}^{2} a^{2} \\
& \approx 2.3 \times 10^{36} \text { photons } / \mathrm{s} .
\end{aligned}
$$

This number should be compared with the number of photons with energies exceeding the ionization energy emitted by the stellar surface per unit time. For the considered temperatures (of the order of $3 \times 10^{4} \mathrm{~K}$ ), this number is

$$
\begin{gathered}
N_{\text {surf }}=4 \pi a^{2} c \frac{k^{3} T_{\mathrm{S}}^{3}}{4 \pi^{2} c^{3} \hbar^{3}}\left(\frac{I_{0}}{k T_{\mathrm{S}}}\right) \exp \left(-\frac{I_{0}}{k T_{\mathrm{S}}}\right) \\
\approx 2 \times 10^{36} \text { photons } / \mathrm{s} .
\end{gathered}
$$

In this case, $N \approx N_{\text {surf }}$. However, our model did not take into account the photons emitted by the spots. For other surface temperatures, $N<N_{\text {surf }}$, since the spot temperatures will be lower.

Our calculations are valid in the absence of polarization of the medium by ionized interstellar hydrogen. In other words, plasma shielding of the electric field is not taken into account. The influence of the plasma becomes substantial when $\operatorname{div} \mathbf{E}<4 \pi \rho(\mathbf{E}$ is 


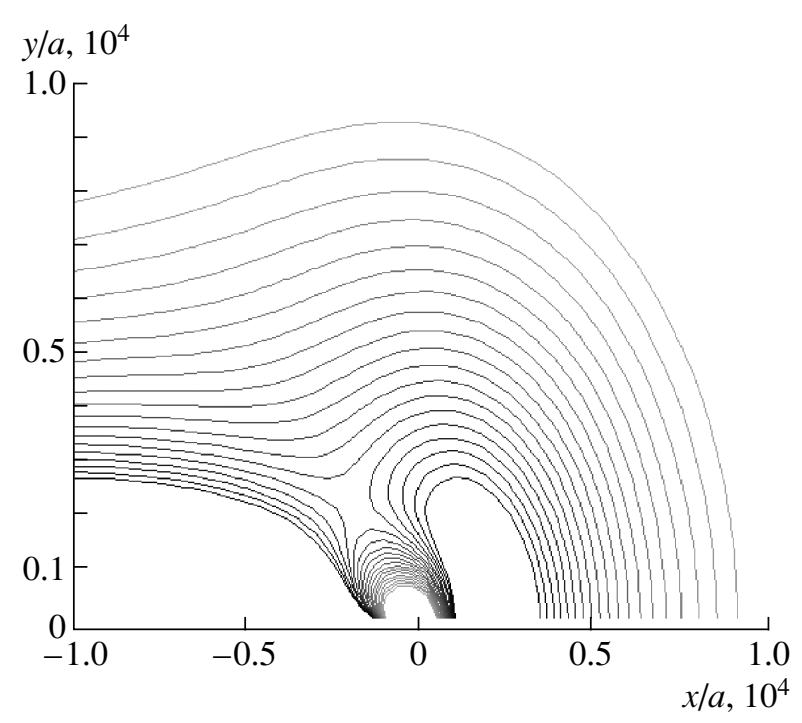

Fig. 3. Same as Fig. 1 for a surface temperature of $0.4 \times 10^{5} \mathrm{~K}$. The ionization rate varies from $0.0098 \mathrm{~cm}^{-3} \mathrm{~s}^{-1}$ to $0.0286 \mathrm{~cm}^{-3} \mathrm{~s}^{-1}$.

the accelerating electric field and $\rho$ the charge density of the ionized interstellar hydrogen). Note that when the charged particles move from the light cylinder towards the surface of the star inside the tube of open field lines, their number density increases as $1 / r^{3}$, while $\operatorname{div} \mathbf{E}$ increases as $1 / r^{1.5}$. As a result, although the polarization of the electric field by plasma can be neglected near the light cylinder, it may be manifest below this region. However, this will not affect the accelerating potential, which depends only on the magnetic field at the light cylinder and the rotational frequency.

As an estimate, the condition of the absence of polarization can be rewritten $\Phi\left(R_{\mathrm{LC}}\right) / R_{\mathrm{LC}}^{2}<$ $4 \pi e \times d N_{\text {ion }} / d t \times R_{\mathrm{LC}} / c$, where $R_{\mathrm{LC}}$ is the radius of the light cylinder, and $d N_{\text {ion }} / d t$ the average ionization rate per unit volume near the light cylinder $\left(\mathrm{cm}^{-3} \mathrm{~s}^{-1}\right)$. For $B_{12}=1, \quad P=1 \mathrm{~s}$, and $n_{\infty \mathrm{H}}=1 \mathrm{~cm}^{-3} T_{\mathrm{S}}=3 \times 10^{4} \mathrm{~K}$ (the temperature corresponding to the most efficient ionization of neutral hydrogen in the region of the light cylinder), the polarization of the medium just begins to be manifest. The polar spot radiates an energy of $\approx 1.9 \times 10^{29} \mathrm{erg} / \mathrm{s}$. For $T_{\mathrm{S}}=4.5 \times 10^{4} \mathrm{~K}$ (the temperature corresponding to the most efficient ionization of neutral helium in the vicinity of the light cylinder (Fig. 7)), $B_{12}=1$, $P=1 \mathrm{~s}$, the polarization of the medium begins to be appreciable only when $n_{\infty \mathrm{H}}=10 \mathrm{~cm}^{-3}$ (i.e., for $\left.n_{\infty \mathrm{He}}=1 \mathrm{~cm}^{-3}\right)$. With these parameters, neutral hydrogen is ionized outside the light cylinder and is then repelled by the magnetic-dipole radiation.

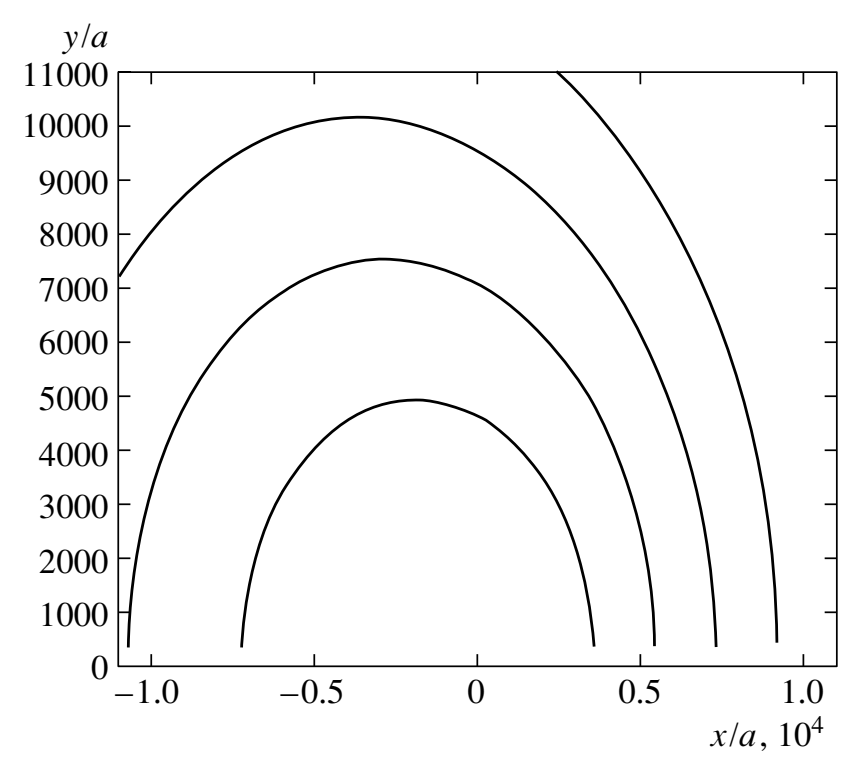

Fig. 4. Same as Fig. 2 for a surface temperature of $0.4 \times 10^{5} \mathrm{~K}$. The fraction of neutrals varies from 0.02 to 0.08 .

In this case, the energy emitted by the spot is also $\approx 1.9 \times 10^{29} \mathrm{erg} / \mathrm{s}$.

These results are valid for both $\boldsymbol{\Omega} \mathbf{m}>0$ and $\mathbf{\Omega} \mathbf{m}<0$ ( $\mathbf{m}$ is the magnetic moment of the star).

In addition to the thermal $\mathrm{X}$-ray radiation of the polar spots, the accretion of neutral gas will also result in the generation of gamma-ray radiation at the light cylinder, due to curvature radiation of the electrons accelerated to relativistic energies. Near the light-cylinder radius, the electric and magnetic fields are appreciably nonorthogonal, resulting in the acceleration of electrons by the electric field along the magnetic-field lines [9]. We will use the vacuum values of the field, without taking into account the Goldreich-Julian magnetosphere, to obtain numerical estimates. For example, for the case of an orthogonal rotator $(\boldsymbol{\Omega} \perp \mathbf{m})$, the electric and magnetic fields on the rotational axis of the rotating magnetic dipole in vacuum for $r \gg a$ are $[4,10]$ :

$$
\begin{gathered}
E=\frac{B_{0}}{2}\left(\frac{\Omega a}{c}\right)^{3} \frac{\sqrt{1+\nu^{2}}}{\nu^{2}}, \\
B=\frac{B_{0}}{2}\left(\frac{\Omega a}{c}\right)^{3} \frac{\sqrt{\nu^{4}-\nu^{2}+1}}{\nu^{3}}, \\
\cos \vartheta=\frac{1}{\sqrt{1+\nu^{6}}},
\end{gathered}
$$

where $\nu=r / R_{\mathrm{LC}}$ and $\vartheta$ is the angle between $\mathbf{E}$ and $\mathbf{B}$. At the light-cylinder radius $(\nu=1)$, $E_{||}=E \cos \vartheta=0.5 B_{0}(\Omega a / c)^{3} \approx 4.6 B_{12} / P^{3}$ cgs. 


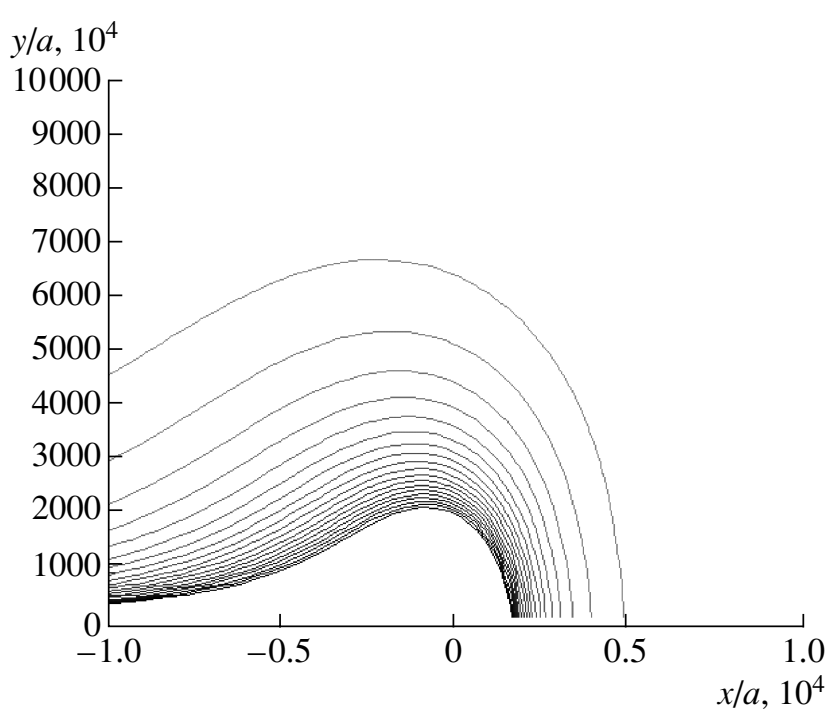

Fig. 5. Same as Fig. 3 for ionization of helium. The ionization rate varies from $0.000824 \mathrm{~cm}^{-3} \mathrm{~s}^{-1}$ to $0.0296 \mathrm{~cm}^{-3} \mathrm{~s}^{-1}$.

The electric field accelerates the electrons along magnetic-field lines up to gamma-factors of

$$
\gamma_{\max }=\frac{e E_{\|} R_{\mathrm{LC}}}{m c^{2}} \approx 1.2 \times 10^{7} \frac{B_{12}}{P^{2}} .
$$

If the acceleration by the electric field is totally balanceed by radiative losses, the equilibrium gammafactor will be

$$
\gamma_{\max }=\left(3 E_{\|} R_{\mathrm{LC}}^{2} / 2 e\right)^{1 / 4} \approx 2.4 \times 10^{7}\left(\frac{B_{12}}{P}\right)^{1 / 4} .
$$

The accelerated electrons move along magneticfield lines whose radius of curvature is of the order of $R_{\mathrm{LC}}$. They can radiate efficiently only at distances from the star that do not appreciably exceed the lightcylinder radius.

The intensity of the radiation of a single electron is

$$
W=\frac{2 e^{2} c \gamma^{4}}{3 R_{\mathrm{LC}}^{2}} \mathrm{erg} / \mathrm{s} .
$$

Averaging the acceleration over the time spent in the vicinity of the light cylinder, we obtain

$$
\langle W\rangle=\frac{1}{5} \frac{2 e^{2} c \gamma_{\max }^{4}}{3 R_{\mathrm{LC}}^{2}} \approx 0.9 \frac{B_{12}}{P^{3}} \mathrm{erg} / \mathrm{s} .
$$

In this average, we assumed that the gamma-factor varies linearly with time.

The characteristic energy of the emitted gammarays is

$\hbar \omega=\frac{\hbar c \gamma_{\max }^{3}}{2 R_{\mathrm{LC}}} \approx 0.9 \times 10^{-5} \frac{B_{12}^{3 / 4}}{P^{7 / 4}} \operatorname{erg} \approx 3 \frac{B_{12}^{3 / 4}}{P^{7 / 4}} \mathrm{MeV}$.

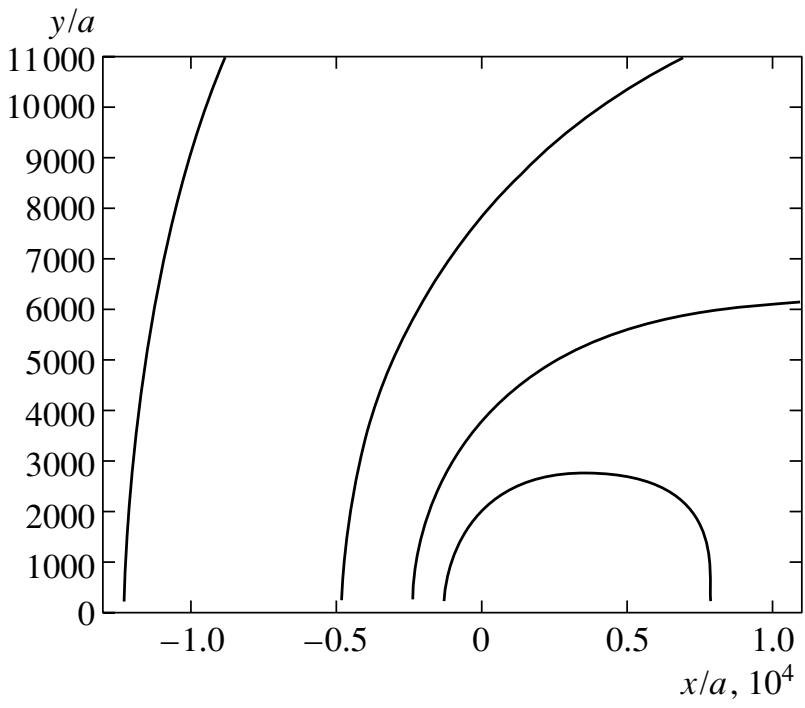

Fig. 6. Same as Fig. 4 for helium neutrals. The fraction of neutrals varies from 0.05 to 0.08 .

We calculated the total intensity of the gamma-ray radiation from the light cylinder using the above accretion model. Figure 8 presents the results of these calculations. The characteristic intensities are of the order of $0.2 \times 10^{29} \mathrm{erg} / \mathrm{s}$.

Note that, if $\boldsymbol{\Omega} \mathbf{m}>0$, gamma-ray radiation will also be generated by relativistic electrons that are ejected from the polar caps due to thermo-emission and then accelerated in the tube of open field lines. The intensity of this radiation can be estimated as $4 \times 10^{29} B_{12}^{2} / P^{4} \mathrm{erg} / \mathrm{s}$, which exceeds the intensity of the gamma-ray radiation due to the accreted neutrals (for $B_{12}=1, P=1 \mathrm{~s}$ ). With the parameters considered, a neutron star may or may not generate radiation at radio wavelengths (depending on the type of nondipolarity of the magnetic field in circumpolar regions [11]). If electron-positron pairs are created in the tube, the reverse positron current will contribute to the $\mathrm{X}$-ray radiation of the polar spots. Observations indicate that the fraction of $\mathrm{X}$-ray radiation in the total magnetic-dipole losses of a pulsar is $10^{-3}$; i.e., of the order of $10^{28} \mathrm{erg} / \mathrm{s}$ for the parameters considered [12]. However, theoretical calculations exceed this value by a factor of a few. In any case, for the parameters considered, the accretion-driven component in the X-ray radiation of the polar spots exceeds the component due to the inverse positron current by an order of magnitude.

\section{ANALYTICAL ESTIMATES \\ OF THE INTENSITY}

\section{OF X-RAY AND GAMMA-RAY RADIATION}

We present below analytical estimates for the above numerical results. The intensity of the gamma-

ASTRONOMYREPORTS Vol.50 No.2 2006 


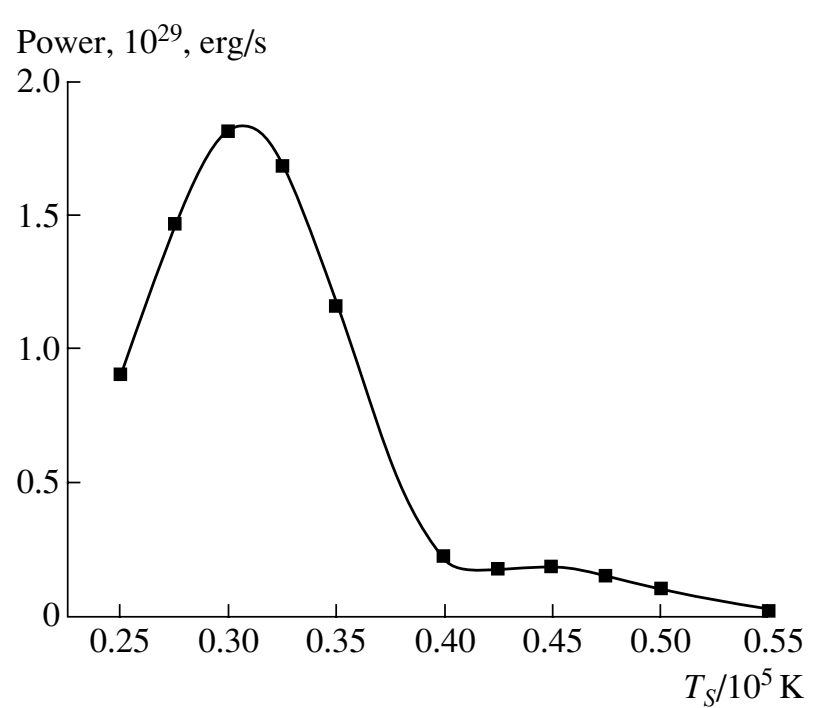

Fig. 7. The energy that goes into heating the spots as a function of the surface temperature of the star, for hydrogen and helium accretion. The first maximum at $T_{\mathrm{S}}=0.3 \times 10^{5} \mathrm{~K}$ corresponds to the ionization of hydrogen, and the second at $T_{\mathrm{S}}=0.45 \times 10^{5} \mathrm{~K}$ to the ionization of helium.

ray radiation is expressed

$$
d E / d t=\langle W\rangle N_{\mathrm{LC}}
$$

Here, $N_{\mathrm{LC}}$ is the number of particles accelerated in the region of the light cylinder:

$$
\begin{aligned}
N_{\mathrm{LC}} & =d N_{\text {ion }} / d t \times 15 R_{\mathrm{LC}}^{3} \frac{R_{\mathrm{LC}}}{c} \\
& =15 n_{\mathrm{ph}} c \sigma_{\mathrm{ph}}\langle n\rangle \frac{R_{\mathrm{LC}}^{4}}{c},
\end{aligned}
$$

where $15 R_{\mathrm{LC}}^{3} \quad$ corresponds to the volume from $0.5 R_{\mathrm{LC}}$ to $1.5 R_{\mathrm{LC}}$, and $\langle n\rangle$ is the average number density of neutrals in the region of the light cylinder. This latter quantity can be estimated from the continuity condition, without taking into account the decrease in the number of neutrals due to ionization:

$$
\langle n\rangle=n_{\infty} \frac{V_{\mathrm{ff}}}{4 V_{\infty}},
$$

where $V_{\mathrm{ff}}=\sqrt{2 G M / R_{\mathrm{LC}}}$ is the free-fall velocity at the light cylinder $\left(V_{\mathrm{ff}} \gg V_{\infty}\right)$.

Thus, we eventually obtain (the numerical value is calculated for abundant "feeding" of hydrogen, i.e., $\left.T_{\mathrm{S}}=3 \times 10^{4} \mathrm{~K}\right)$

$$
\begin{aligned}
d E / d t= & 0.9 \frac{B_{12}}{P^{3}} \times 15 n_{\mathrm{ph}} c \sigma_{\mathrm{ph}} \frac{\sqrt{2 G M}}{4 V_{\infty}} \frac{R_{\mathrm{LC}}^{3.5}}{c} \\
& \approx 0.1 \times 10^{29} \frac{B_{12}}{P^{1.5}} \mathrm{erg} / \mathrm{s} .
\end{aligned}
$$

Power, $10^{29}, \mathrm{erg} / \mathrm{s}$

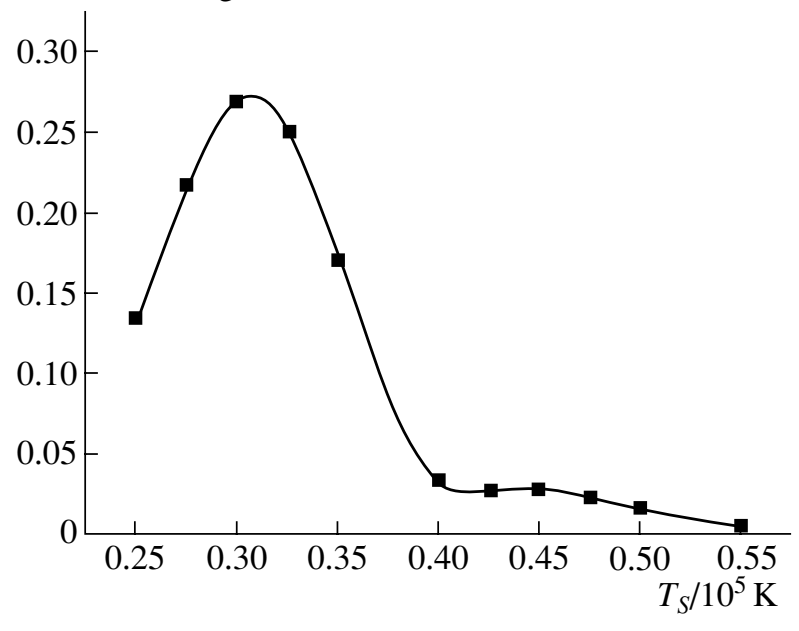

Fig. 8. The intensity of the gamma-ray radiation from the light cylinder as a function of the surface temperature for hydrogen and helium accretion. The first maximum at $T_{\mathrm{S}}=0.3 \times 10^{5} \mathrm{~K}$ corresponds to the ionization of hydrogen, and the second at $T_{\mathrm{S}}=0.45 \times 10^{5} \mathrm{~K}$ to the ionization of helium.

We can obtain the intensity of the $\mathrm{X}$-ray radiation from the polar caps in the same way $\left(T_{\mathrm{S}}=3 \times 10^{4} \mathrm{~K}\right)$

$$
d E / d t=1.5 \times 10^{29} \frac{B_{12}}{P^{3}} \mathrm{erg} / \mathrm{s},
$$

which is consistent with our model calculations.

\section{THE PULSAR J0826+2637}

It is possible that $\mathrm{X}$-ray radiation due to the accretion of neutral hydrogen or helium has already been observed. One example is the radio and X-ray pulsar J0826+2637, with a period of $P=0.53$ s, magnetic field of $B=9.64 \times 10^{11} \mathrm{G}$, and age of $4.92 \times 10^{6} \mathrm{yr}$. The temperature $T=100 \mathrm{eV}$ and radius $R=0.56 \mathrm{~km}$ of its polar cap were derived from its $\mathrm{X}$-ray spectrum. Thus, the intensity of the radiation from the spot is $10^{30} \mathrm{erg} / \mathrm{s}$. The magnetic-dipole losses are $3.4 \times 10^{32} \mathrm{erg} / \mathrm{s}$; i.e., the X-ray radiation of the polar spots due to the reverse positron current should be of the order of $3 \times 10^{29} \mathrm{erg} / \mathrm{s}$.

Calculations for the X-ray intensity due to accretion yield the following results. With a surface temperature of $T_{\mathrm{S}} \approx 3 \times 10^{4} \mathrm{~K}$, hydrogen feeding is abundant and the intensity reaches $5.5 \times 10^{29} \mathrm{erg} / \mathrm{s}$ (for a number density of interstellar hydrogen of $1 \mathrm{~cm}^{-3}$ ). When $T_{\mathrm{S}} \approx 4 \times 10^{4} \mathrm{~K}$, abundant helium feeding yields the same intensity for a number density of interstellar hydrogen of $10 \mathrm{~cm}^{-3}$. The observed radiation may be due to both the reverse positron current and the accretion of neutral interstellar gas. 


\section{RESULTS}

We have shown that the accretion of neutral hydrogen and helium can affect the X-ray and gammaray radiation of cool neutron stars. Our model for a neutron star with $B_{12}=1$ and $P=1 \mathrm{~s}$ indicates that the magnetosphere is fed most efficiently when the surface temperature of the star is $T_{\mathrm{S}} \approx 3 \times 10^{4} \mathrm{~K}$ for hydrogen feeding or $T_{\mathrm{S}}=4.5 \times 10^{4} \mathrm{~K}$ for helium feeding. For a number density of interstellar hydrogen of $1 \mathrm{~cm}^{-3}$, the temperature of the polar spots reaches $1.5 \times 10^{6} \mathrm{~K}$, while their X-ray luminosity is $2 \times 10^{29} \mathrm{erg} / \mathrm{s}$. Even if electron-positron plasma is being formed vigorously into the tube, the reverse positron current is not able to provide such a high luminosity. The intensity of the gamma-ray radiation of the ionized interstellar gas can reach $0.2 \times 10^{29} \mathrm{erg} / \mathrm{s}$. However, if the angle between the magnetic moment and the angular velocity of rotation of the star is acute, the radiation will not be seen against the $4 \times 10^{29} \mathrm{erg} / \mathrm{s}$ background of the primary electron beam.

In conclusion, we note that the energy released due to the accretion of neutral hydrogen or helium exceeds $m c^{2}$ (where $m$ is the mass of the accreted matter) by three orders of magnitude. Therefore, to obtain an X-ray impulse with a duration of $30 \mathrm{~s}$ and an intensity of $4 \times 10^{29} \mathrm{erg} / \mathrm{s}$, it is sufficient that 10 tons of neutral hydrogen or helium fall onto the neutron star.

\section{ACKNOWLEDGMENTS}

This work was supported by the Russian Foundation for Basic Research (project no. 04-02-17590), the Federal Science and Technology Project in Astronomy (project no. 40.002.1.1.1103), the Program in Support of Leading Scientific Schools of the Russian Federation (grant no. NSh-1115.2003.2), and the Dynasty Foundation for noncommercial programs.

\section{REFERENCES}

1. V. M. Lipunov, Astrophysics of Neutron Stars (Nauka, Moscow, 1987) [in Russian].

2. A. I. Tsygan, Pis'ma Astron. Zh. 3, 531 (1977) [Astron. Lett. 3, 289 (1977)].

3. G. A. E. Wright, Nature 280, 40, 41 (1979).

4. A. I. Tsygan, Astron. Zh. 60, 960 (1983) [Sov. Astron. 27, 555 (1983)].

5. D. Page, in Neutron Stars and Pulsars, Ed. by N. Shibazaki, N. Kawai, S. Shibata, and T. Kifune (1997), p. 183.

6. D. Harding, R. A. Guyer, and G. Greenstein, Astrophys. J. 222, 991 (1978).

7. L. D. Landau and E. M. Lifshitz, Mechanics, 3rd ed. (Nauka, Moscow, 1973; Pergamon Press, Oxford, 1976).

8. J. Arons and E. T. Scharlemann, Astrophys. J. 231, 854 (1979).

9. A. Ferrari and E. Trussoni, Astron. Astrophys. 36, 267 (1974).

10. A. Deutch, Ann. Astrophys. 18, 1 (1955).

11. E. M. Kantor and A. I. Tsygan, Astron. Zh. 81, 1 (2004) [Astron. Rep. 48, 1029 (2004)].

12. W. Becker and J. Trumper, NATO ASI Ser. 515, 525 (1996).

Translated by K. Maslennikov 